\title{
Self-assembly kinetics of amphiphilic dendritic copolymers
}

Article

Accepted Version

Zhang, C., Fan, Y., Zhang, Y., Yu, C., Li, H., Chen, Y., Hamley, I. W. and Jiang, S. (2017) Self-assembly kinetics of amphiphilic dendritic copolymers. Macromolecules, 50 (4). pp. 1657-1665. ISSN 0024-9297 doi: https://doi.org/10.1021/acs.macromol.6b02331 Available at https://centaur.reading.ac.uk/70405/

It is advisable to refer to the publisher's version if you intend to cite from the work. See Guidance on citing.

Published version at: http://dx.doi.org/10.1021/acs.macromol.6b02331

To link to this article DOI: http://dx.doi.org/10.1021/acs.macromol.6b02331

Publisher: American Chemical Society

All outputs in CentAUR are protected by Intellectual Property Rights law, including copyright law. Copyright and IPR is retained by the creators or other copyright holders. Terms and conditions for use of this material are defined in the End User Agreement.

\section{www.reading.ac.uk/centaur}

\section{CentAUR}

Central Archive at the University of Reading 
Reading's research outputs online 


\section{Self-assembly kinetics of amphiphilic dendritic polymers}

Cuiyun Zhang ${ }^{123}$, You Fan ${ }^{5}$, Yunyi Zhang ${ }^{123}$, Cong Yu ${ }^{23}$, Hongfei $\mathrm{Li}^{34}$, Yu Chen ${ }^{5}$, Ian William Hamley $^{6}$, Shichun Jiang ${ }^{1}$

${ }^{1}$ School of Materials Science and Engineering, Tianjin University, Tianjin 300072, P.

R. China

${ }^{2}$ State Key Laboratory of Electroanalytical Chemistry, Changchun Institute of Applied Chemistry, Chinese Academy of Sciences, Changchun 130022, P. R. China ${ }^{3}$ University of Chinese Academy of Science, Beijing, 100049, P. R. China ${ }^{4}$ State Key Laboratory of Polymer Physics and Chemistry, Changchun Institute of Applied Chemistry, Chinese Academy of Sciences, Changchun 130022, P. R. China

${ }^{5}$ Department of Chemistry, School of Sciences, Tianjin University, Tianjin 300072, P.

R. China

${ }^{6}$ School of Chemistry, Pharmacy and Food Biosciences, University of Reading, Whiteknights, Reading RG6 6AD, United Kingdom 
ABSTRACT: The self-assembly process of amphiphilic dendritic copolymers (ADPs) with a hydrophilic core and hydrophobic shell was investigated via laser light scattering. The self-assembly occurs via a fast step and a slow step with different relaxation times. At the critical micelle concentration (CMC), the fusion of small micelles results in the rapid increase of the micelle size in the fast step. The slow step is associated with equilibrium through the fission and fusion of the micelles. The micelle size increases with the unimer concentration, which leads to a lower micelle concentration. The lower micelle concentration and larger micelle size make the relaxation time of the fast step increase with increasing unimer concentration. The fusion of the larger micelles at higher concentration is more efficient for the increase of the micelle size, which contributes to the decrease of relaxation time in the slow step. Moreover, the fusion of small micelles with large micelles at higher concentration accelerates the approaching equilibrium of the micelle except for the fission and fusion of micelles. With the increasing degree of amidation (DA), the relaxation time in the fast step increases and in the slow step it decreases.

\section{INTRODUCTION}

Following the linear, branched, crosslinking polymers, dendritic polymers including hyperbranched polymers and dendrimers are the fourth-generation of polymer architectures. ${ }^{1,2}$ Hyperbranched polymers consisting of dendritic, linear, and terminal units have irregular structure compared with dendrimers. Dendritic polymers are highly branched with a three-dimensional architecture and have low viscosity and better solubility than the linear analogues. Great efforts have been devoted to 
understand and control the self-assembly of amphiphilic dendritic polymers. Multiple morphologies of self-assembled dendritic polymers, such as micelles, vesicles, macroscopic tubes and fibers have been reported ${ }^{3-6}$ and the self-assembly has been exploited in areas of drug delivery, sensors and cell imaging, etc. ${ }^{7-9}$

The self-assembled morphology of polymers is influenced by their topology, the solvent quality and the solvophilic/solvophobic ratio. For the amphiphilic polymers containing a hyperbranched core, they can self-assemble into spherical micelles, wormlike micelles and vesicles with an increase in the degree of branching of the core. ${ }^{10}$ The self-assembled structure of a gradient copolymer was observed to change from spherical micelles to cylindrical micelles and then to vesicles with the worsening solvent quality. ${ }^{11}$ The size of the spherical micelles formed from amphiphilic hyperbranched polymers in aqueous solution decreases with increaseing hydrophilic volume fraction. ${ }^{12,13}$ In 2005, Yan suggested a multimicelle aggregate mechanism (MMA), which included two steps, the dendritic polymers first self-assembled into small micelles and then the small micelles aggregated to form large micelles. ${ }^{12}$ In 2007, Yan and Haag observed small spherical building units inside the micelles by TEM and cro-TEM, ${ }^{3,14}$ which proved the MMA mechanism. Based on dissipative particle dynamics simulations, the dynamic self-assembly processes and the detailed self-assembly mechanism were analyzed, which demonstrated the unimolecular micelle aggregate mechanism and the small micelle aggregate mechanism. In the former mechanism, the unimolecular micelles directly aggregate to form large micelles without microphase separation. In the small micelle aggregate mechanism, 
the amphiphilic dendritic polymers first rearrange the hydrophilic and hydrophobic parts to form microphase-separated small micelles. Then the small micelles assemble into large multimolecular micelles. Also the increase of the radius of gyration $\left(R_{\mathrm{g}}\right)$ with time reflects the dynamic process. ${ }^{15}$ However, except for computer simulations, the dynamic self-assembly process of dendritic polymers is rarely reported. The fusion process of polymer vesicles was observed by optical microscopy. ${ }^{16,17}$ But the formation process of the vesicles before the size is large enough for the observation by optical microscopy is unclear.

The micellization kinetics of surfactants and block copolymers have been studied both theoretically and experimently. ${ }^{18-22}$ The Aniansson and Wall theory provides a widely accepted kinetic description of the self-assembly process of small surfactant molecules, which divides the micellization process into a fast and a slow step. ${ }^{18,21}$ In the fast step, the aggregation number $\left(N_{\mathrm{agg}}\right)$ of each micelle increases with no change in the total number of micelles. In the slow step, the self-assembly process approaches equilibrium accompanied with the formation and deformation of the micelles with a variation in the number of micelles. The micellization process of block polymers is similar to that of surfactants. The unimers quickly associate to form quasi-equilibrium micelles and the increasing micelle number dominates over the growth of the micelles in the first process. In the second process the micellization process approaches equilibrium with an increase in micelle size combined with a decrease in the number of micelles. ${ }^{22}$ The self-assembly kinetics of the dendritic polymers is expected to be similar. 
To date, the study of the self-assembly of dendritic polymers mainly focused on the final equilibrium structure which was observed by TEM. ${ }^{3-6}$ During the sample preparation, the solvent evaporation leads to shrinkage, collapse, and even deformation of the micelles. ${ }^{23}$ The increasing concentration resulting from the solvent evaporation may induce the fusion of micelles, which creates artefacts in TEM images. In contrast, it is possible to measure $R_{\mathrm{g}}$ and hydrodynamic radius $\left(R_{\mathrm{h}}\right)$ in situ using laser light scattering. Furthermore, the time dependence of scattering intensity, $R_{\mathrm{g}}$ and $R_{\mathrm{h}}$ provide information on the dynamic processes of self-assembly.

In this paper, first, the critical self-assembly condition of the dendritic polymers was explored. Second, the dynamic self-assembly process was studied. Then the influence of concentration and solvophilic/solvophobic ratio on the self-assembly kinetic process was discussed. We believe that this represents the first time the self-assembly kinetics of dendritic polymers by using laser light scattering method.

\section{EXPERIMENTAL SECTION}

2.1 Materials. Hyperbranched polyethylenimine (HPEI, $M_{\mathrm{n}}=10^{4} \mathrm{~g} \mathrm{~mol}^{-1}, M_{\mathrm{n}} / M_{\mathrm{w}}=$ 2.5) was purchased from Aldrich. 2,2-Bis(hydroxymethyl)propionic acid (BHP, 99\%) was purchased from Beijing Ouhe Technology Company. Palmitic acid (A.R.) was purchased from Tianjin University Kewei Chemical Company. The amphiphilic dendritic copolymers with a HPEI core and a dendritic palmitate tail shell were synthesized according to the reported approach. ${ }^{24}$ Detailed information on molar mass and $R_{\mathrm{h}}$ of the studied amphiphilic dendritic polymers (ADPs) ${ }^{25}$, as well as CMC and critical solvent volume fraction (CSV) values are listed in Tables 1 . The structure of 
the dendritic polymers was shown in Figure S1. Chloroform and methanol used in the self-assembly experiment were purchased from the Beijing chemical works (Beijing, China). Chloroform was refluxed with calcium hydride before distillation.

2.2 Measurements. The light scattering experiments were performed on ALV CGS-3 light scattering spectrometer equipped with an ALV-5000 multi-tau digital time correlator and a He-Ne laser $(\lambda=632 \mathrm{~nm})$ as light source. The $R_{\mathrm{g}}$ values of the micelles was calculated from the angular dependence of the natural logarithm of the scattered intensity according to the Guinier plot. ${ }^{26}$ The decay rate distribution $(\mathrm{G}(\Gamma))$ was obtained from the CONTIN Laplace inversion of the intensity-intensity time correlation function $\mathrm{g}^{2}(q, \tau)$. The relation between the decay rate and the apparent diffusion coefficient is $\Gamma=q^{2} D$, where the scattering vector $q=(4 \pi n / \lambda) \sin (\theta / 2), \mathrm{n}$ is the refractive index of the solvent, $\lambda$ is the wavelength of the light in vacuum, and $\theta$ is the scattering angle. ${ }^{27,28}$ From the Stokes-Einstein equation $R_{\mathrm{h}}=k_{\mathrm{B}} T / 6 \pi \eta D$, the average hydrodynamic radius was calculated, where $k_{\mathrm{B}}, T$ and $\eta$ are the Boltzmann constant, the absolute temperature and the solvent viscosity, respectively. The dependence of the laser light scattering intensity on the angle was performed in the range of $30^{\circ}$ to $90^{\circ}$, every $10^{\circ}$. All the solutions were filtered through $0.45 \mu \mathrm{m}$ Millipore polytetrafluoroethylene filters before measurements.

Table 1: Structure parameters and the critical self-assembly condition for the ADPs

$\begin{array}{llllll}\text { Polymer } & \mathrm{DA} & M_{\mathrm{n}} / 10^{4} & R_{\mathrm{h}} / \mathrm{nm} & \mathrm{CSV} & \mathrm{CMC} /\left(\mathrm{mg}^{*} \mathrm{ml}^{-1}\right) \\ \text { P-1 } & 38 \% & 6.2 & 5.2 & 49 \% & 0.57 \\ \text { P-2 } & 41 \% & 6.7 & 5.5 & 47 \% & 0.42 \\ \text { P-3 } & 51 \% & 8.0 & 6.1 & 44 \% & 0.26 \\ \text { P-4 } & 60 \% & 9.3 & 7.3 & 44 \% & 0.20\end{array}$




\section{RESULTS AND DISCUSSION}

3.1 The critical self-assembly condition for the ADPs. ADPs were first dissolved in the common solvent (chloroform) and then the non-solvent (methanol) for the non-polar shell was added dropwise to change the solvent quality gradually. The values of $\mathrm{CSV}$ that the ADPs started to self-assemble were obtained from the scattering intensity of the solution. The final concentration of the ADPs was $0.5 \mathrm{mg} / \mathrm{ml}$ and before measurement the solutions was kept at $25{ }^{\circ} \mathrm{C}$ for $12 \mathrm{~h}$. As shown in Figure 1, at the CSV, the scattering intensity increases sharply upon the formation of micelles. The solvophilic/solvophobic ratio increases with DA, which leads to decreasing polarity of the ADPs. The CSV decreases slightly with the DA (Table1). In terms of the CSV of P-1, P-2, P-3 and P-4, the solvent containing 50\% methanol is a relatively poor solvent and the ADPs self-assemble in solvent mixtures containing this fraction of methanol.

The CMC for the four ADPs was measured in solutions that contained $50 \%$ methanol. At the $\mathrm{CMC}$, the scattering intensity of the solution increases due to the formation of micelles. In Figure 2, the scattering intensity of P-1 increases at a concentration of $0.57 \mathrm{mg} / \mathrm{ml}$, suggesting that the CMC for P-1 is $0.57 \mathrm{mg} / \mathrm{ml}$. As shown in Table 1, the CMC for the ADPs decreases with increasing DA, because of the increase of the solvophobic/solvophilic ratio with DA. 


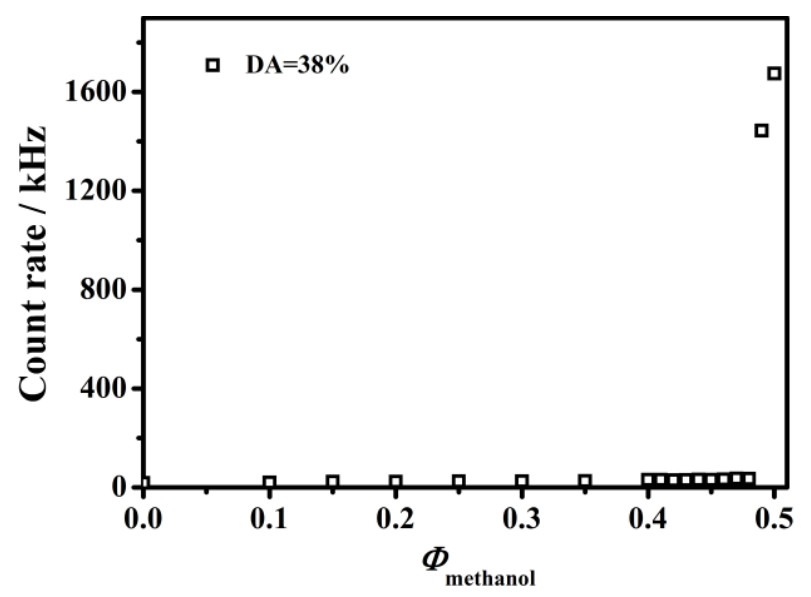

Figure 1. Scattering intensity vs methanol volume fraction for polymer P-1.

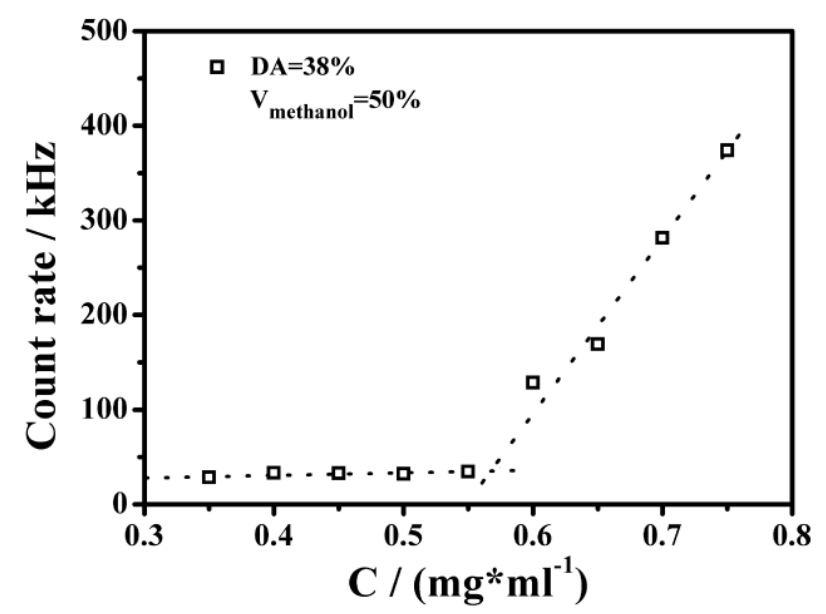

Figure 2. Scattering intensity vs concentration for polymer P-1(C is the concentration). 

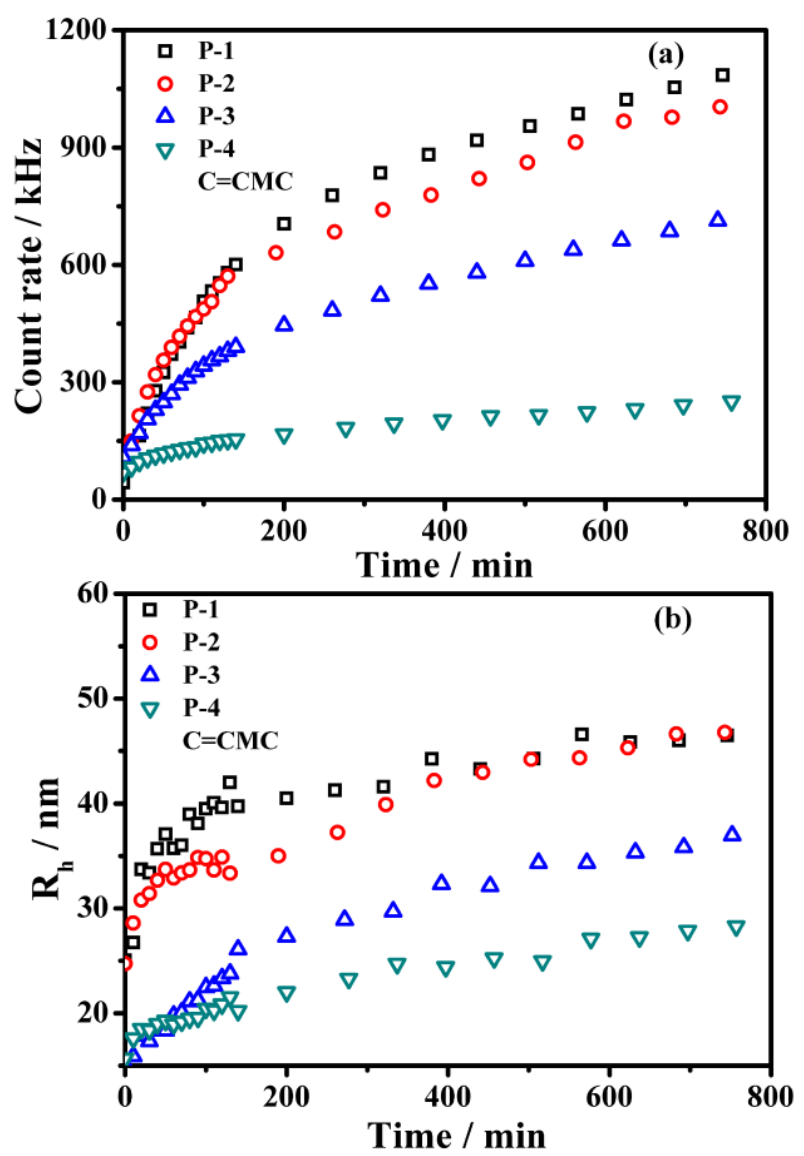

Figure 3. (a) The time-dependence of scattering intensity. (b) The time-dependence of the hydrodynamic radius.

3.2 The self-assembly kinetics of ADPs. To date, to our knowledge, there are no reported experimental studies on the self-assembly kinetics of ADPs. In this work, the real-time self-assembly process was monitored and discussed. The time dependence of the scattering intensity and $R_{\mathrm{h}}$ were measured. The increasing trend of the scattering intensity (Figure $3 \mathrm{a}$ ) and $R_{\mathrm{h}}$ (Figure $3 \mathrm{~b}$ ) are almost the same, with a rapid initial increase followed by a slower increase. Figure 4a represents the scattering vector dependent decay rate and scattering intensity. Based on the Stocks-Einstein equation and the Guinier plot, $R_{\mathrm{h}}$ and $R_{\mathrm{g}}$ values were calculated. For spherical micelles, $R_{\mathrm{h}}$ is independent of scattering angle. ${ }^{29}$ The ratio of $R_{\mathrm{g}}$ and $R_{\mathrm{h}}$ reflects the 
morphology of the aggregates, being 1.5 for a random coil, 1 for a vesicle and 0.77 for a micelle. ${ }^{30,31}$ Figure $4 \mathrm{~b}$ shows that the ratio of $R_{\mathrm{g}}$ and $R_{\mathrm{h}}$ for P-1, P-2, P-3, P-4 at the CMC is around 0.75 , indicating that the self-assembled structure is micelles. The micelle size observed by TEM for P-1 and P-2 at CMC are larger than that measured by laser light scattering, which is caused by the fusion of micelles during the sample preparation. The TEM images of P-3 and P-4 agree well with the laser light scattering. (Figure S2)

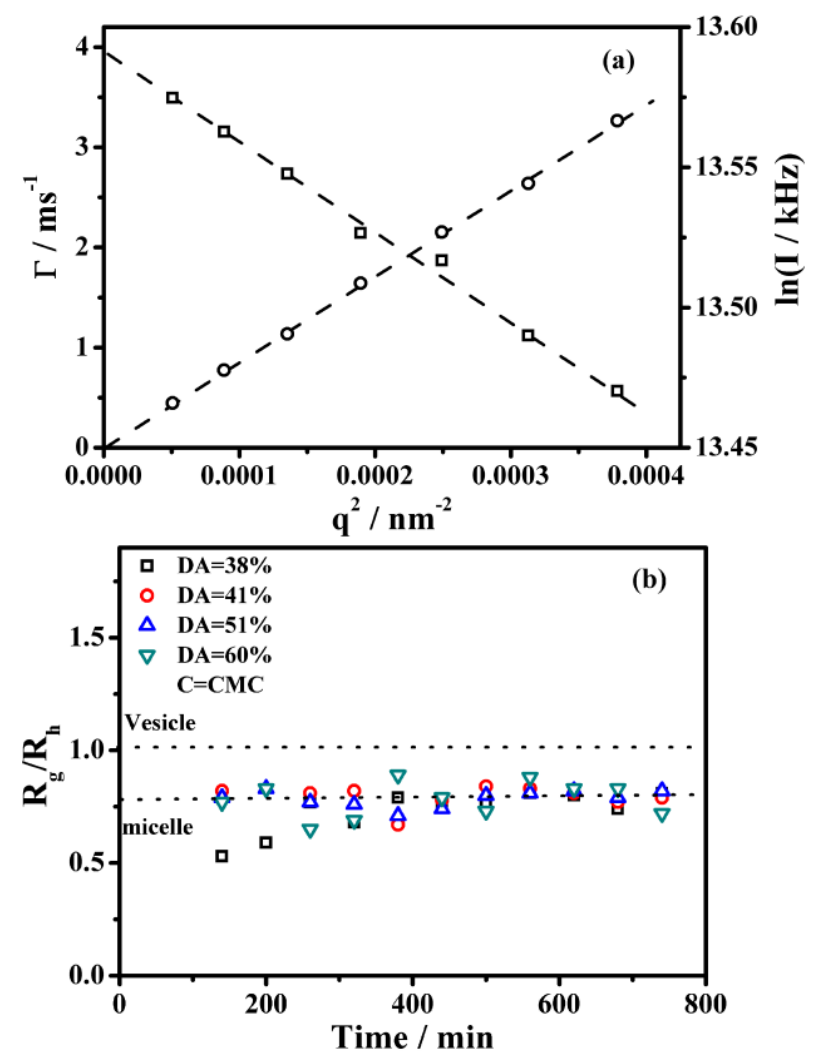

Figure 4. (a) Scattering vector dependence of the average decay rate ( $\square$ ) and scattering intensity

(०) for P-1. (b) The time related ratio of $R_{\mathrm{g}}$ and $R_{\mathrm{h}}$ for P-1, P-2, P-3and P-4.

According to the self-assembly of surfactants and block copolymers, ${ }^{18,20}$ the kinetics of the dendritic copolymers was analyzed based on the time dependence of 
the scattering intensity, which was best fitted using the double exponential function (eq. 1).

$$
\mathrm{y}=y_{0}+a\left(1-\exp \left(-t / \tau_{1}\right)+b\left(1-\exp \left(-t / \tau_{2}\right)\right)\right.
$$

Here, $\tau_{1}$ and $\tau_{2}$ are relaxation times for the two processes, $y_{0}, a$ and $b$ are adjustable parameters related to the self-assembly condition. The term $y_{0}$ represents the initial scattering intensity. $a$ and $b$ represent the contribution of the two steps to the self-assembly process. The correlation coefficients for all the fitting results are above 0.99, which indicates the high quality of the fitting and the precision of the kinetic parameters. As shown in Table 2, the two relaxation times correspond to the two different self-assembly steps. The high DA of the dendritic polymers leads to great steric hindrance, ${ }^{25}$ which hinders the microphase separation of the unimer in the self-assembly. Because of the compact structure and the great steric hindrance, the self-assembly is assumed to follow the unimolecular micelle aggregate mechanism. ${ }^{15}$ Based on eq. 3, the $N_{\text {agg }}$ of the micelle was estimated. $V_{\text {micelle }}$ and $V_{\text {unimer }}$ are the volume of the micelle and the unimer, which are obtained from eq. 2. The rapid increase of $N_{\text {agg }}$ corresponds to the first step and the slow increase of $N_{\text {agg }}$ occurs in the second step of the self-assembly (Figure 5). The necessary condition for the self-assembly is that the chemical potential of the unimers in the micelles is smaller than that in the solution and when the chemical potential of the unimers in the micelle holds a minimum value, the process reaches equilibrium. ${ }^{32}$

$$
V=\frac{4}{3} \pi R^{3}
$$




$$
N_{\text {agg }}=\frac{V_{\text {micelle }}}{V_{\text {unimer }}}
$$

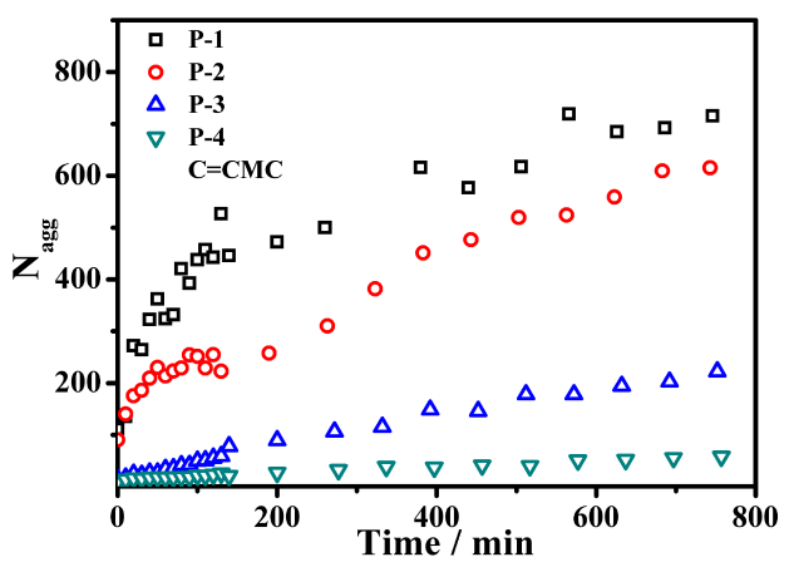

Figure 5. Variation of the $N_{\text {agg }}$ with time.
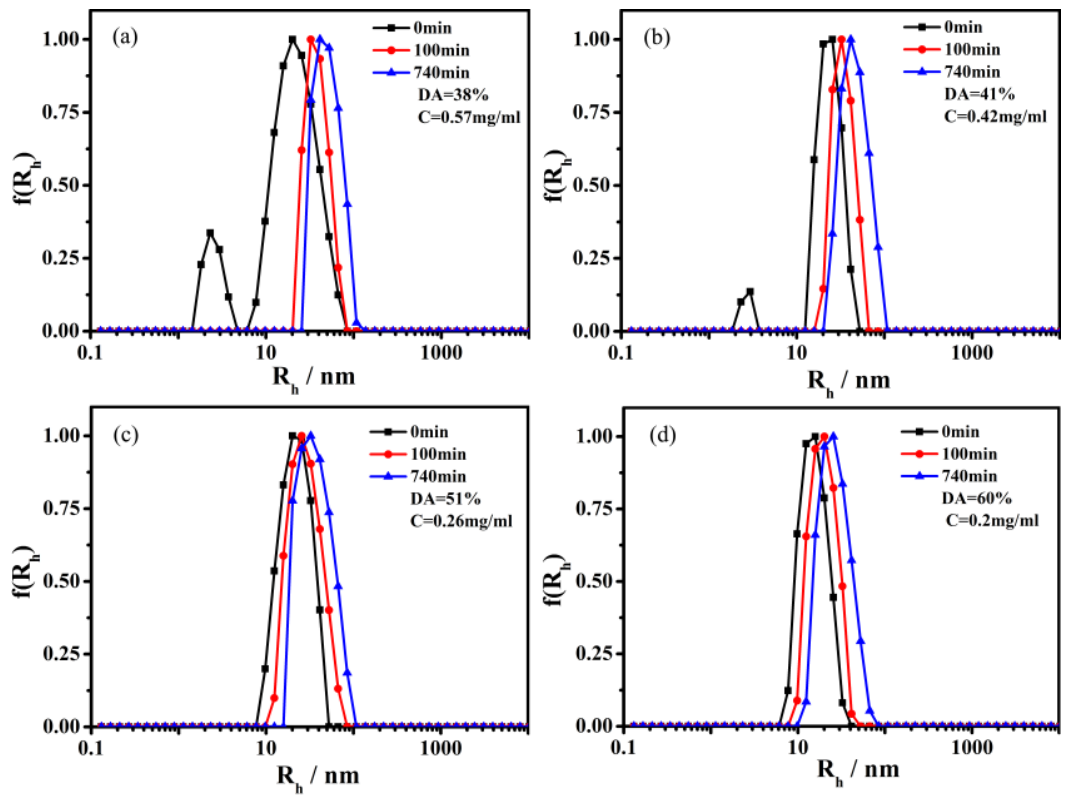

Figure 6. The distribution functions of the apparent hydrodynamic radius of P-1, P-2, P-3, P-4 at CMC.

The kinetics of self-assembly are reflected by the increase of $R_{\mathrm{h}}$. After addition of methanol, the ADPs self-assemble into small micelles and with time the small micelles grow into larger ones. The distribution of $R_{\mathrm{h}}$ at the CMC is shown in Figure 6. Unfortunately, because of the fast association of unimers, only for P-1 and P-2 can the 
self-assembly process of the unimer to micelle transition be monitored owing to the the relatively small solvophobic/solvophilic ratio. Due to the lack of data on the unimer to micelle transition, we mainly focus on the fusion and fission of the micelles. There are two ways for the increase of micelle size. One is the fusion of the small micelles. The other is the fission and fusion occurred among the micelles. In order to illustrate the self-assembly kinetics more obviously, the increased hydrodynamic radius $\left(R_{\mathrm{h}}\right)$ was calculated based on eq.4,

$$
V_{i+1}=V_{i}+V_{I}
$$

where $V$ is the volume of the micelles. $V_{\mathrm{i}}$ and $V_{\mathrm{i}+1}$ are the volumes of the micelles at the times $i$ and $i+1 . V_{I}$ is the increased volume. Through the values of $V_{\mathrm{I}}$, the value of $R_{\mathrm{h} \text { I }}$ was calculated. The evolution of $R_{\mathrm{h}}$ and the smallest hydrodynamic radius of the micelles in the distribution $\left(R_{\mathrm{h} \text { min }}\right)$ are shown in Figure 7 . The dotted line in Figure 7 is the cut-off line between the first and the second step. In the second step, the value of $R_{\mathrm{h}}$ is smaller than $R_{\mathrm{h} \text { min }}$ and this suggests that the micelles formed in the first step fission and fuse with each other, which cause the slow increase of the micelle size. Before 80 minutes (min) the polydispersity index (PDI) obtained from the ratio of $\mu_{2} /$ $\Gamma^{2}\left(\mu_{2}\right.$ is the second cumulant) decreases with the time and increases after $80 \mathrm{~min}$ (Figure. 8). From the evolution of $R_{\mathrm{h}}, R_{\mathrm{h}}$ and the change of PDI, the self-assembly process of the ADPs at the CMC is summarized. In the first step, the unimers aggregate quickly to form small micelles and then the small micelles fuse into relatively larger and monodisperse micelles. In the second step, through the fission and fusion of the micelles the distribution becomes broader and the self-assembly 
approaches equilibrium.

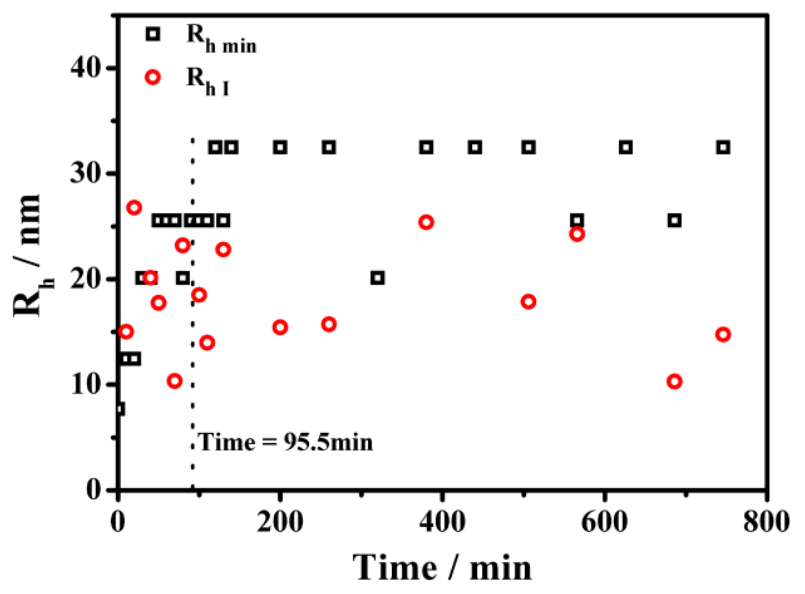

Figure 7. The increase of the hydrodynamic radius of the smallest micelles in the distribution and the increased hydrodynamic radius of $\mathrm{P}-1$ with time at $\mathrm{CMC}$.

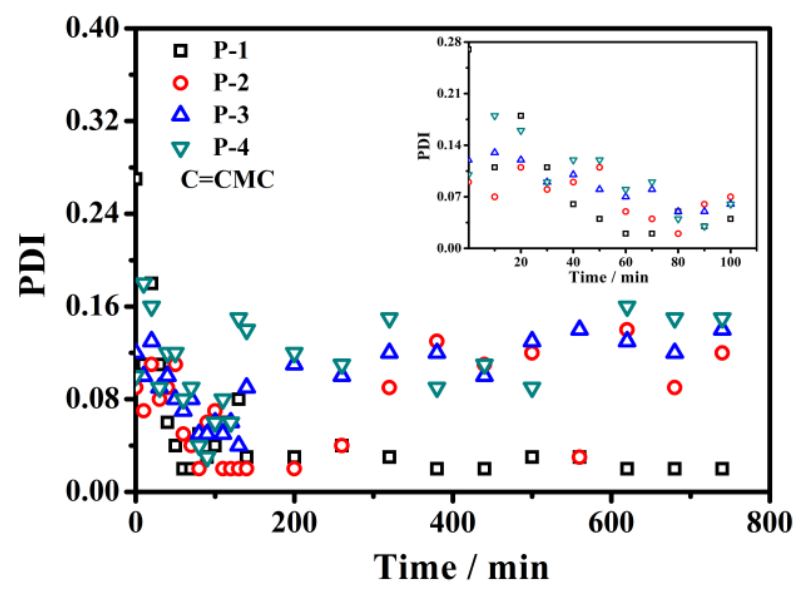

Figure 8. Time dependence of the polydispersity index.

3.3 Influence of concentration on the self-assembly. The phase diagram of the block polymers is influenced by the concentration ${ }^{33,34}$ and the size of the nanoparticles formed from the self-assembly of the hyperbranched polymers is larger at higher concentration $^{35}$. As shown in Figure 9, the scattering intensity and hydrodynamic radius increase with concentration. The relaxation time of the self-assembly at different concentration is shown in Table 2. With increasing concentration, $\tau_{1}$ increases and $\tau_{2}$ decreases. Honda et al. reported that the relaxation time $\tau_{1}$ of block 
copolymer in the first process decreased with increasing concentration and $\tau_{2}$ was almost independent of the concentration. ${ }^{22}$ However, the relaxation times of block copolymers in the rod to vesicle transition increase with increasing concentration. ${ }^{20}$
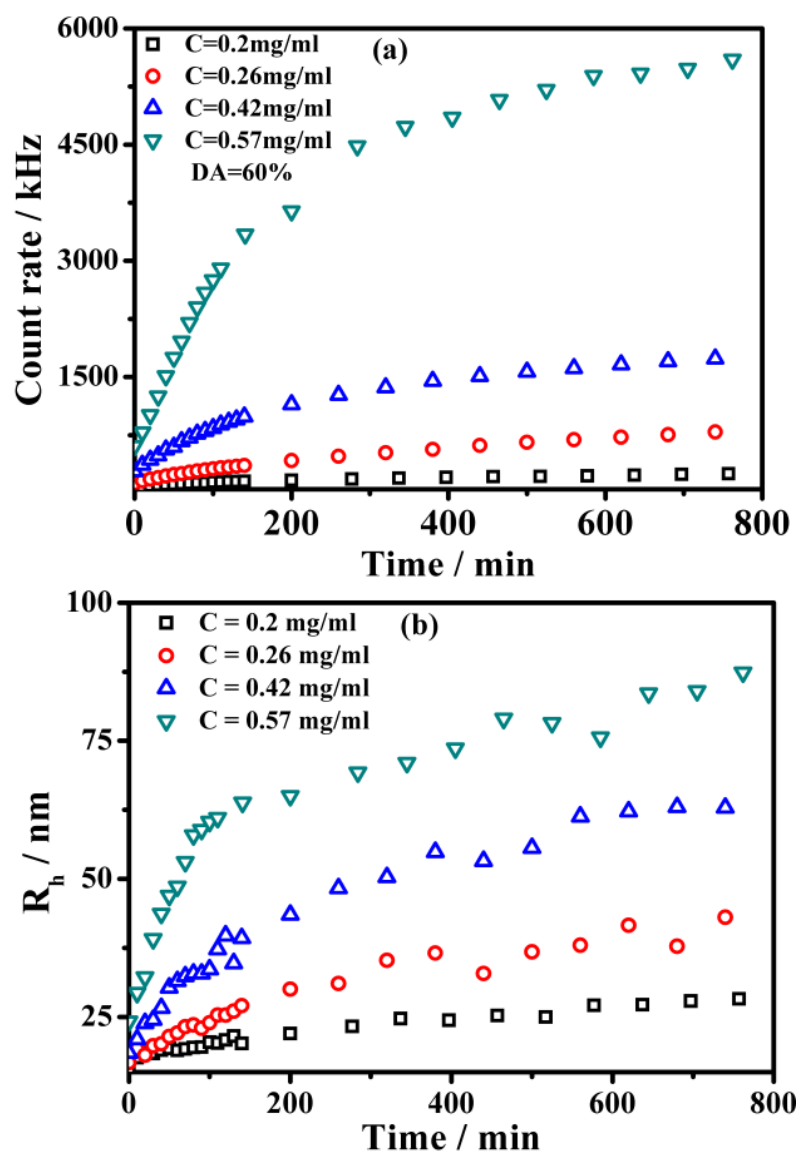

Figure 9. Time-dependent related scattering intensity (a) and hydrodynamic radius (b) with fixed concentration for polymer P-4.

Table 2. Kinetic results for the ADPs at different concentration

$\begin{array}{ccccccc}\text { DA } & C / \mathrm{mg}^{*} \mathrm{ml}^{-1} & y_{0} / \mathrm{kHz} & a / \mathrm{kHz} & \tau_{1} / \mathrm{min} & b / \mathrm{kHz} & \tau_{2} / \mathrm{min} \\ 61 \% & 0.2 & 71.6 & 59.5 & 53.9 & 241.9 & 1143.9 \\ & 0.26 & 129.5 & 86.9 & 52.0 & 987.9 & 856.5 \\ & 0.42 & 283.7 & 647.7 & 110.3 & 1140.3 & 602.6 \\ 51 \% & 0.57 & 504.3 & 3441.1 & 129.8 & 2367.6 & 636.6 \\ & 0.26 & 105.4 & 247.7 & 77.6 & 1193.7 & 2067.1 \\ & 0.42 & 133.6 & 635.3 & 69.2 & 2151.9 & 1033 \\ 41 \% & 0.57 & 288.6 & 2378.4 & 125.1 & 2826.4 & 751.1 \\ & 0.42 & 84.3 & 403.4 & 59.8 & 1625.1 & 1891.2 \\ & 0.57 & 195.3 & 647.7 & 91.1 & 1201.8 & 846.1\end{array}$


The first step of the micellization of surfactant and block copolymer is mainly the quick association of the unimers and the relaxation time is on the time scale of seconds. ${ }^{18,22}$ Whereas, in this study, the first step of ADPs is influenced by the fusion of the small micelles and the relaxation time is on the time scale of hours. The energy barrier for the fusion of micelle is larger than that for the aggregation of unimers, which suggests that different mechanisms lead to the difference in the time scale. The fusion of micelles is concentration dependent and at higher micelle concentration the collision opportunity increases. ${ }^{36-38}$ The increase of hydrodynamic radius with concentration may result in the decrease of the small micelle concentration in the first step. The self-assembly rate will slow down for the decrease of small micelle concentration. The concentration ratio of two solutions is expressed as high concentration to low concentration. For the two solutions of a given ADP with different concentrations, if the $N_{\text {agg }}$ ratio is larger than the corresponding unimer concentration ratio, then the micelle concentration of the solution with a higher unimer concentration must be lower, and vice versa. As shown in Figure 10a, the $N_{\text {agg }}$ ratio is larger than the ratio of the concentration, indicating the decrease of the micelle concentration with increasing unimer concentration. Also, the fusion energy barrier increases with the micelle size, which suggests that the fusion of the large micelles is unfavourable. ${ }^{39}$ The increasing value of $\tau_{1}$ with concentration in the first step results from the decreasing micelle concentration and the rising fusion energy barrier of the large micelles. The derivative of the change of scattering intensity with time is shown 
in Figure 10b. The $Y$-axis value of the derivative reflects the increasing rate of the scattering intensity. The increasing rate in the first step is larger than in the second step. Though at higher concentration the relaxation time increases, the increasing rate of the micelle size in the first step increases. The larger micelle size at higher concentration leads to more efficient fusion.

The polydispersity index and $R_{\mathrm{h}}$ increase with the concentration (Figure S3). For P-4 with a concentration of $0.57 \mathrm{mg} / \mathrm{ml}$, the distribution of the hydrodynamic radius even shows a bimodal distribution after 100min (Figure S4). With increasing concentration, the self-assembly kinetics in the second step becomes complex. In the second step, the increased hydrodynamic radius is larger than the minimum hydrodynamic radius in the distribution (Figure 11). Except for the fission and fusion of the micelles, the self-assembly in the second step includes the fusion of the small micelles with the large micelles. The size of the small micelles increases at higher concentration (Figure S5). The larger increasing rate of scattering intensity at higher concentration means that the micelles formed in the first step are closer to the equilibrium size and so the micelles in the second step quickly approach the equilibrium size. This contributes to the decrease of $\tau_{2}$ with increasing concentration. 

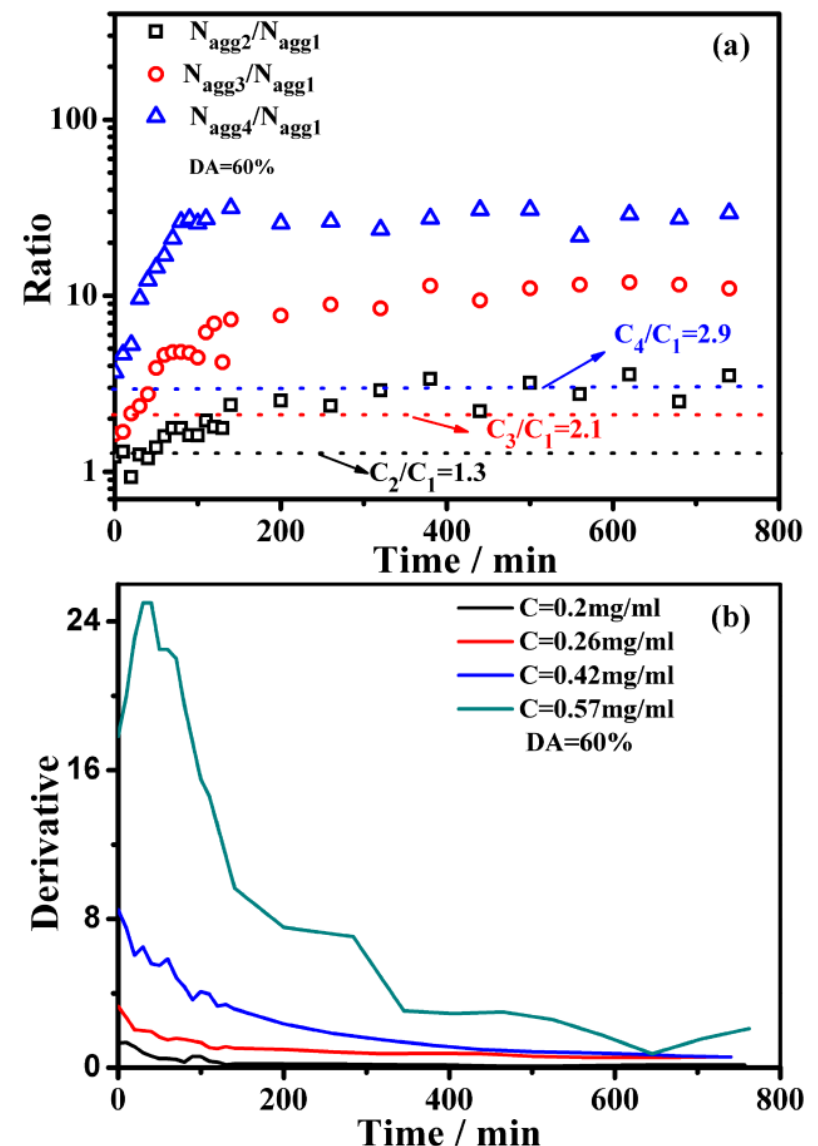

Figure 10. (a) The comparison of the concentration ratio and the $N_{\text {agg }}$ ratio at different concentrations for polymer P-4. $\left(\mathrm{C}_{2} / \mathrm{C}_{1}\right.$ represents $(0.26 \mathrm{mg} / \mathrm{ml}) /(0.2 \mathrm{mg} / \mathrm{ml}) . \mathrm{C}_{3} / \mathrm{C}_{1}$ represents $(0.42 \mathrm{mg} / \mathrm{ml}) /(0.2 \mathrm{mg} / \mathrm{ml}) . \mathrm{C}_{4} / \mathrm{C}_{1}$ represents $(0.57 \mathrm{mg} / \mathrm{ml}) /(0.2 \mathrm{mg} / \mathrm{ml}) . N_{\text {agg }} / N_{\text {aggl }}$ represents the $N_{\text {agg }}$ ratio of the micelle in the solution with a concentration of $0.26 \mathrm{mg} / \mathrm{ml}$ and $0.2 \mathrm{mg} / \mathrm{ml} . N_{\text {agg } 3} / N_{\text {agg } 1}$ represents the $N_{\text {agg }}$ ratio of the micelle in the solution with a concentration of $0.42 \mathrm{mg} / \mathrm{ml}$ and $0.2 \mathrm{mg} / \mathrm{ml} . N_{\text {agga }} / N_{\text {agg1 }}$ represents the $N_{\text {agg }}$ ratio of the micelle in the solution with a concentration of $0.57 \mathrm{mg} / \mathrm{ml}$ and $0.2 \mathrm{mg} / \mathrm{ml}$.) (b) The derivative of the change of scattering intensity with time at different concentration. 


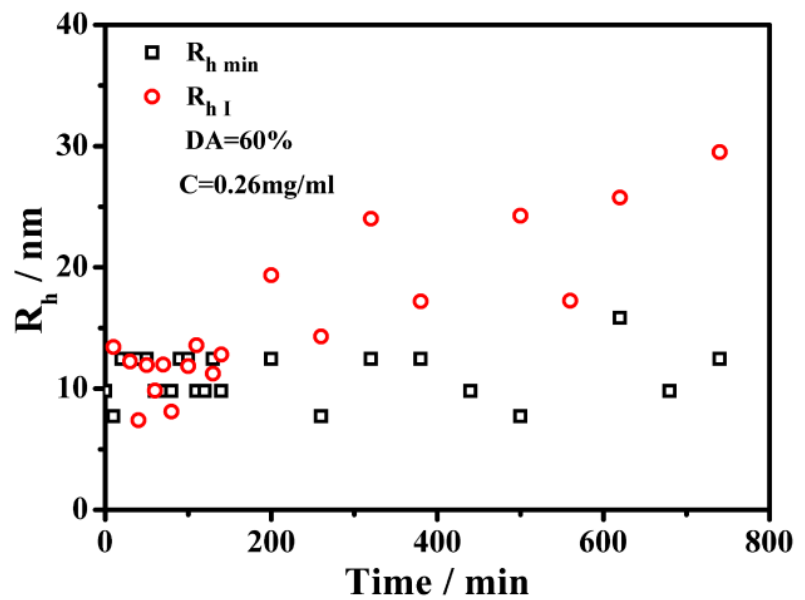

Figure 11. The increase of the smallest micelle hydrodynamic radius in the distribution and the increased hydrodynamic radius of P-4 with the time.

3.4 Influence of the DA on the self-assembly. Liu et al. ${ }^{40}$ reported that the size and $N_{\text {agg }}$ of the spherical micelles increased with the solvophobic chain length of linear block copolymer. The micellization mechanism of the slow process changes from unimer insertion/expulsion to micelle fusion/fission, because of the decreasing apparent activation energy with the increasing solvophobic chain length. The micelle of the hyperbranched copolymer is also influenced by the solvophilic/solvophobic ratio and the degree of branching of the core. ${ }^{4,41}$ For the ADPs, the absence of the microphase separation resulting from the great steric hindrance of the non-polar shell is conjectured to make the micellization kinetics different. As shown in Figure12, the scattering intensity and the hydrodynamic radius increase with DA. The ratio of the radius of gyration and hydrodynamic is around 0.74 (Figure S6), indicating that the structure is not influenced by DA. 

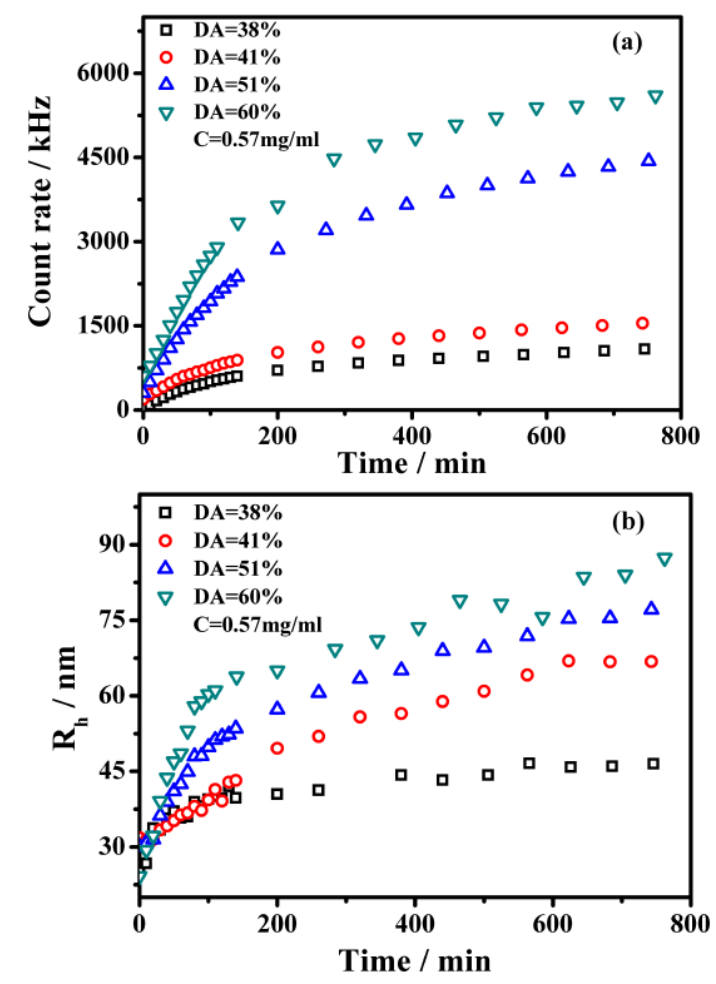

Figure 12. Time dependent scattering intensity (a) and hydrodynamic radius (b) with DA.

A study of the aggregation kinetics of block copolymer found that the driving force for the micellization increased with the solvophobic chain length, giving rise to the decreasing relaxation time. ${ }^{40}$ Similar to such block copolymers, the self-assembly driving force of the ADPs is assumed to increase with the increasing DA. As shown in Table 2, with the increasing DA, $\tau_{1}$ increases and $\tau_{2}$ decreases at the same concentration. The molecular weight increases with DA, which induces the decrease of the unimer molar concentration. This phenomenon may cause the increase of $\tau_{1}$. However, as illustrated in the influence of concentration, with the increasing unimer concentration, the micelle concentration decreases and the relaxation time in the first step increases, which suggests that the increase of the unimer molar concentration will not lead to the decrease of $\tau_{1}$. The $N_{\text {agg }}$ of the four ADPs is almost the same in the first step, while the $N_{\text {agg }}$ of P-1 is much smaller than that of the other three ADPs in the 
second step (Figure 13a). Despite the larger $R_{\mathrm{h}}$ of the micelle, the $N_{\mathrm{agg}}$ of P-4 is slightly smaller than that of the P-2 and P-3 (Figure 13a). In order to analyze the micelle concentration of the four ADPs in the micellization process, the comparison of the $N_{\text {agg }}$ ratio and the unimer molar concentration ratio for different ADP solutions is shown in Figure $13 \mathrm{~b}, \mathrm{c}$, d. If the $N_{\text {agg }}$ ratio is larger than the unimer molar concentration ratio, the micelle concentration will be smaller at higher DA, and vice versa. Before the intersection point for the $N_{\text {agg }}$ ratio and the molar concentration ratio $\left(T_{1}\right)$, the $N_{\text {agg }}$ ratio is smaller than the unimer molar concentration, suggesting that the micelle concentration is larger at higher DA. After $T_{1}$, the $N_{\text {agg }}$ ratio is larger than the unimer molar concentration ratio, indicating that the micelle concentration is smaller at higher DA. $T_{1}$ is smaller than $\tau_{1}$ (Table 3 ), which indicates that the smaller micelle concentration in the later period of the first step has an influence on the self-assembly kinetics. The increasing $\tau_{1}$ results from the larger micelle size and the smaller micelle concentration, which is similar to the influence of concentration on the kinetics.
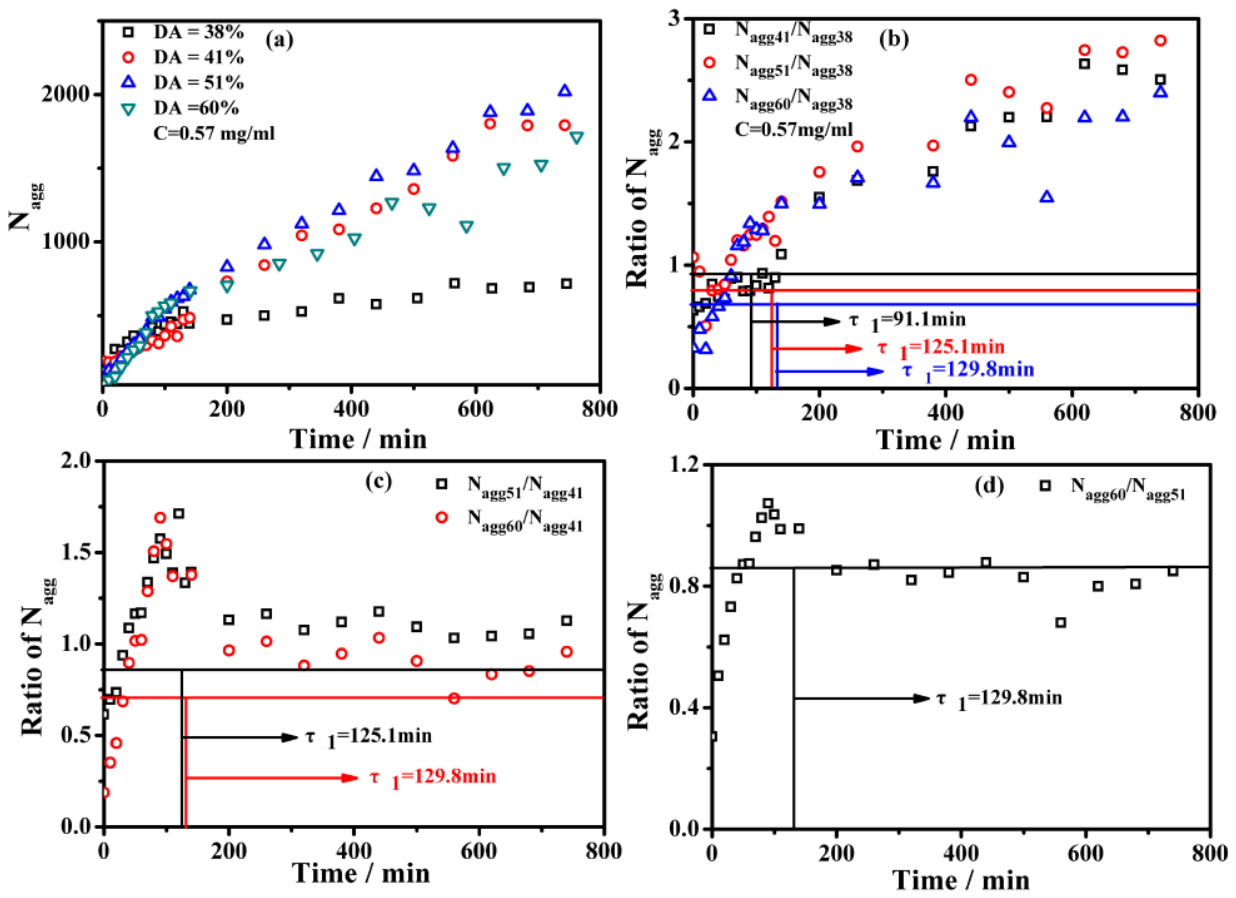
Figure 13.(a) The change of $N_{\text {agg }}$ with the time for P-1, P-2, P-3, P-4 at $0.57 \mathrm{mg} / \mathrm{ml}$. (b) The comparison of the $N_{\text {agg }}$ ratio and unimer molar concentration ratio for different ADPs at $0.57 \mathrm{mg} / \mathrm{ml}$. (The subscript number of the $N_{\mathrm{agg}}$ represents the DA. The solid line is the corresponding molar concentration ratio).

Table 3. The comparsion of $T_{1}$ and the relaxation time $\tau_{1}$

$\begin{array}{lllllll}\text { Ratio } & N_{\text {agg41 }} / N_{\text {agg38 }} & N_{\text {agg51 }} / N_{\text {agg38 }} & N_{\text {agg60 }} / N_{\text {agg38 }} & N_{\text {agg51 }} / N_{\text {agg41 }} & N_{\text {agg60 }} / N_{\text {agg41 }} & N_{\text {agg60 }} / N_{\text {agg51 }} \\ T_{1} / \text { min } & 100 & 46 & 46 & 30 & 30 & 49 \\ \tau_{1} / \text { min } & 91.1 & 125.1 & 129.8 & 125.1 & 129.8 & 129.8\end{array}$

The derivative of the change of the scattering intensity with time is shown in Figure 14. The $Y$-axis value of the derivative increases with DA. Because the micelle size increases with DA, in the first step, the fusion rate of micelles slows down, while the rate of size increase is larger, indicating that the fusion is more efficient for the increase of the micelle size. Except for P-1, with increasing DA, the concentration of the ADPs solution is higher than the CMC, meaning that the kinetics of P-2, P-3 and P-4 in the second step includes the fusion of the small micelles with the large micelles. The size of the micelles that fuse the big micelles in the second step increases with increasing DA (Figure S7). Based on these two aspects, the micelle in the second step approaches the equilibrium size more quickly at higher DA and the relaxation time $\tau_{2}$ decreases with DA. In the second step, because of the larger $N_{\text {agg }}$ ratio compared with the unimer molar concentration ratio for P-2, P-3 and P-4 with respect to P-1 (Figure $13 \mathrm{~b}$ ), the micelle concentration is much smaller than that of $\mathrm{P}-1$, while $\tau_{2}$ for $\mathrm{P}-1$ is much larger than that of P-2, P-3 and P-4 (Table 2). This phenomenon results from the weaker driving force for P-1 compared with ADPs with higher DA. 


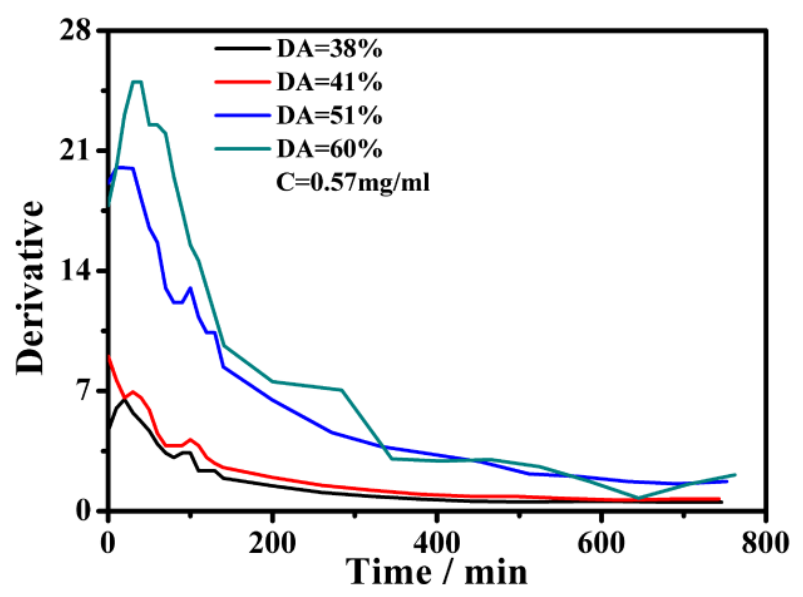

Figure 14. The derivative of the change of scattering intensity with time at $0.57 \mathrm{mg} / \mathrm{ml}$ for P-1, P-2, P-3, P-4.

\section{Conclusion}

Adding a poor solvent for the dendritic non-polar shell induces the self-assembly of ADPs in solution and the self-assembly is time dependent. From the evolution of scattering intensity and hydrodynamic radius with time, the self-assembly process of the ADPs was analyzed. The kinetics is influenced by the concentration. At the CMC, the small micelles associate with each other to form narrowly distributed micelles in the first step. In the following step, $R_{\mathrm{h}}$ is smaller than $R_{\mathrm{h} \text { min }}$, indicating that fusion of micelles of comparable size does not occur, and the increase of the micelle size is caused by the fission and fusion of micelles when collision occurs. This phenomenon make the micelle distribution become broader in the second step. Through the fission and fusion of the large micelles, the self-assembly finally approaches dynamic equilibrium. Distinct to the behavior of surfactants and amphiphilic block copolymers, the relaxation time of the first step increases with increasing concentration. Compared with the unimer concentration ratio, the $N_{\text {agg }}$ ratio is larger, which induces the smaller micelle concentration at higher unimer concentration. On the other side, the 
increasing fusion energy barrier with increasing micelle size makes the fusion of large micelle slower. Due to the smaller micelle concentration and the slower fusion rate, the value of $\tau_{1}$ increases with the unimer concentration. The value of the derivative for the change of scattering intensity with time is larger at higher concentration, suggesting that in spite of the slow fusion rate of the large micelles, the increasing rate of the micelle size is faster at higher concentration. The efficient fusion at higher concentration accelerates the approaching equilibrium of the micelle in the second step. Except for the fission and fusion, the second step contains the fusion of small micelles with large micelles at higher unimer concentration. Based on the above, $\tau_{2}$ decreases with increasing concentration. The self-assembly kinetics is also influenced by the solvophobic/solvophilic ratio. The values of $R_{\mathrm{h}}$ increase with increasing DA. However, the values of $N_{\text {agg }}$ are almost the same with increasing DA in the first step and in the following step the $N_{\text {agg }}$ of P-2, P-3 and P-4 is similar, but is larger than that of P-1. Because of the decreasing micelle concentration in the later stage and the larger fusion energy barriers at higher concentration, $\tau_{1}$ increases with increasing DA. The same with the influence of concentration, because of the more efficient fusion the micelle, $\tau_{2}$ decreases with the increasing DA. To the best of our knowledge, this is the first time that the detailed self-assembly kinetics of ADPs has been studied, and our results provide detailed insights into aggregation mechanisms.

\section{ASSOCIATED CONTENT}

\section{Supporting Information}

The Supporting Information is available free of charge on the ACS Publications 
website

\section{AUTHOR INFORMATION}

Corresponding Authors

*E-mail: congyu@ciac.ac.cn

*E-mail: hfli@ciac.ac.cn

*E-mail: chenyu@ tju.edu.cn

*E-mail: $\underline{\text { i.w.hamley@ reading.ac.uk }}$

*E-mail: scjiang@tju.edu.cn

\section{Notes}

The authors declare no competing financial interest.

\section{Ackonwledgments}

This work was supported by the National Nature Science Foundation of China (21374077 and 51573131), the Open Funds of the State Key Laboratory of Electroanalytical Chemistry (SKLEAC201505). The Royal Society (UK) is thanked for an International Collaboration Grant (ref. IE140849).

\section{REFERENCES}

1. Jiang, W.; Zhou, Y.; Yan, D. Hyperbranched Polymer Vesicles: from Self-assembly, Characterization, Mechanisms, and Properties to Applications. Chem. Soc. Rev. 2015, 44, 3874-3889.

2. Zhang, D.; Xu, Z.; Li, J.; Chen, S.; Cheng, J.; Zhang, A.; Chen, S.; Miao, M. Self-Assembly of Amido-Ended Hyperbranched Polyester Films with a Highly Ordered Dendritic Structure. ACS Appl. Mater. Interfaces 2014, 6, 16375-16383. 
3. Hong, H.; Mai, Y.; Zhou, Y.; Yan, D.; Cui, J. Self-Assembly of Large Multimolecular Micelles from Hyperbranched Star Copolymers. Macromol. Rapid Commun. 2007, 28, 591-596.

4. Zhou, Y.; Yan, D. Supramolecular Self-Assembly of Giant Polymer Vesicles with Controlled Sizes. Angew. Chem. Int. Ed. 2004, 43, 4896-4899.

5. Yan, D.; Zhou, Y.; Hou, J. Supramolecular Self-Assembly of Macroscopic Tubes. Science 2004, 303, 65-67.

6. Ornatska, M.; Peleshanko, S.; Genson, K. L.; Rybak, B.; Bergman, K. N.; Tsukruk, V. V. Assembling of Amphiphilic Highly Branched Molecules in Supramolecular Nanofibers. $J$. Am. Chem. Soc. 2004, 126, 9675-9684.

7. Prabaharan, M.; Grailer, J. J.; Pilla, S.; Steeber, D. A.; Gong, S. Folate-Conjugated Amphiphilic Hyperbranched Block Copolymers Based on Boltorn H40, Poly(L-lactide) and Poly(ethylene glycol) for Tumor Targeted Drug Delivery. Biomaterials 2009, 30, 3009-3019.

8. Shi, H.; Chen, X.; Liu, S.; Xu, H.; An, Z.; Ouyang, L.; Tu, Z.; Zhao, Q.; Fan, Q.; Wang, L.; Huang, W. Hyper-Branched Phosphorescent Conjugated Polyelectrolytes for Time-Resolved Heparin Sensing. ACS Appl. Mater. Interfaces 2013, 5, 4562-4568.

9. Yu, S.; Dong, R.; Chen, J.; Chen, F.; Jiang, W.; Zhou, Y.; Zhu, X.; Yan, D. Synthesis and Self-Assembly of Amphiphilic Aptamer-Functionalized Hyperbranched Multiarm Copolymers for Targeted Cancer Imaging. Biomacromolecules 2014, 15, 1828-1836.

10. Wang, J.; Wang, X.; Yang, F.; Shen, H.; You, Y.; Wu, D. Effect of Topological Structures on the Self-Assembly Behavior of Supramolecular Amphiphiles. Langmuir 2015, 31, $13834-13841$. 
11. Zheng, C.; Huang, H.; He, T. Micellization of St/MMA Gradient Copolymers: A General Picture of Structural Transitions in Gradient Copolymer Micelles. Macromol. Rapid Commun. 2013, 34, 1654-1661.

12. Mai, Y. Y.; Zhou, Y. F.; Yan, D. Y. Synthesis and Size-Controllable Self-Assembly of a Novel Amphiphilic Hyperbranched Multiarm Copolyether. Macromolecules 2005, 38, 8679-8686.

13. Du, W.; Li, Y.; Nystrom, A. M.; Cheng, C.; Wooley, K. L. Synthesis, Characterization, and Aqueous Self-Assembly of Amphiphilic Poly(ethylene oxide)-Functionalized Hyperbranched Fluoropolymers. J. Polym. Sci., Part A: Polym. Chem. 2010, 48, 3487-3496.

14. Radowski, M. R.; Shukla, A.; von Berlepsch, H.; Böttcher, C.; Pickaert, G.; Rehage, H.; Haag, R. Supramolecular Aggregates of Dendritic Multishell Architectures as Universal Nanocarriers. Angew. Chem. Int. Ed. 2007, 46, 1265-1269.

15. Wang, Y.; Li, B.; Zhou, Y.; Lu, Z.; Yan, D. Dissipative Particle Dynamics Simulation Study on the Mechanisms of Self-Assembly of Large Multimolecular Micelles from Amphiphilic Dendritic Multiarm Copolymers. Soft Matter 2013, 9, 3293-3304.

16. Zhou, Y.; Yan, D.; Dong, W.; Tian, Y. Temperature-Responsive Phase Transition of Polymer Vesicles: Real-Time Morphology Observation and Molecular Mechanism. J. Phys. Chem. B 2007, 111, 1262-1270.

17. Mai, Y.; Zhou, Y.; Yan, D. Real-Time Hierarchical Self-Assembly of Large Compound Vesicles from an Amphiphilic Hyperbranched Multiarm Copolymer. Small 2007, 3, $1170-1173$.

18. Aniansson, E. A. G.; Wall, S. N.; Almgren, M.; Hoffmann, H.; Kielmann, I.; Ulbricht, W.; Zana, R.; Lang, J.; Tondre, C. Theory of the Kinetics of Micellar Equilibria and Quantitative 
Interpretation of Chemical Relaxation Studies of Micellar Solutions of Ionic Surfactants. $J$. Phys. Chem. 1976, 80, 905-922.

19. Jensen, G. V.; Lund, R.; Gummel, J.; Monkenbusch, M.; Narayanan, T.; Pedersen, J. S. Direct Observation of the Formation of Surfactant Micelles under Nonisothermal Conditions by Synchrotron SAXS. J. Am. Chem. Soc. 2013, 135, 7214-7222.

20. Chen, L.; Shen, H.; Eisenberg, A. Kinetics and Mechanism of the Rod-to-Vesicle Transition of Block Copolymer Aggregates in Dilute Solution. J. Phys. Chem. B 1999, 103, 9488-9497.

21. Iyama, K.; Nose, T. Kinetics of Micelle Formation with Change of Micelle Shape in a Dilute Solution of Diblock Copolymers. Macromolecules 1998, 31, 7356-7364.

22. Honda, C.; Hasegawa, Y.; Hirunuma, R.; Nose, T. Micellization Kinetics of Block Copolymers in Selective Solvent. Macromolecules 1994, 27, 7660-7668.

23. Zhao, H.; Chen, Q.; Hong, L.; Zhao, L.; Wang, J.; Wu, C. What Morphologies Do We Want? -TEM Images from Dilute Diblock Copolymer Solutions. Macromol. Chem. Phys. 2011, 212, 663-672.

24. Liu, Y.; Fan, Y.; Liu, X.-Y.; Jiang, S.-Z.; Yuan, Y.; Chen, Y.; Cheng, F.; Jiang, S.-C. Amphiphilic Hyperbranched Copolymers bearing a Hyperbranched Core and Dendritic Shell: Synthesis, Characterization and Guest Eencapsulation Performance. Soft Matter 2012, 8, 8361-8369.

25. Zhang, C.; Yu, C.; Lu, Y.; Li, H.; Chen, Y.; Huo, H.; Hamley, I. W.; Jiang, S. Hydrodynamic Behaviors of Amphiphilic Dendritic Polymers with Different Degrees of Amidation. Polym. Chem. 2016, 7, 3126-3133.

26. Gunier A., Fournet G. small angle scattering of X-rays, John Wiley \& Sons Inc: New York, 
1955.

27. Wu, C.; Xia, K. Q. Incorporation of a Differential Refractometer into a Laser Light-Scattering Spectrometer. Rev. Sci. Instrum. 1994, 65, 587-590.

28. Chu, B.; Lin, F. L. Laser Light Scattering Study of a Ternary Liquid Mixture: Ethanol-Water-Chloroform. J. Chem. Phys. 1974, 61, 5132-5146.

29. Mountrichas, G.; Mpiri, M.; Pispas, S. Micelles of Star Block (PSPI) 8 and PSPI Diblock Copolymers $(\mathrm{PS}=$ Polystyrene, PI = Polyisoprene): Structure and Kinetics of Micellization . Macromolecules 2005, 38, 940-947.

30. Tu, Y. F.; Wan, X. H.; Zhang, D.; Zhou, Q. F.; Wu, C. Self-Assembled Nanostructure of a Novel Coil-Rod Diblock Copolymer in Dilute Solution. J. Am. Chem. Soc. 2000, 122, 10201-10205.

31. Huo, H.; Li, K.; Wang, Q.; Wu, C. Self-Assembly and Optical Property of Triblock Copolymers Made of Polystyrene and Oligo( $p$-phenyleneethynylene) in Different Mixtures of Toluene and Hexane. Macromolecules 2007, 40, 6692-6698.

32. Israelachvili J. N. Intermolecular and Surface Forces. 3rd ed.; Elsevier: USA, 2011.

33. Shen, H. W.; Eisenberg, A. Morphological Phase Diagram for a Ternary System of Block Copolymer $\mathrm{PS}_{310}-b$-PAA $52 /$ Dioxane/ $\mathrm{H}_{2}$ O. J. Phys. Chem. B 1999, 103, 9473-9487.

34. Hamley, I. W. Block Copolymers in Solution: Fundamentals and Applications. John Wiley \& Sons, Ltd: UK, 2005.

35. Hatton, F. L.; Chambon, P.; McDonald, T. O.; Owen, A.; Rannard, S. P. Hyperbranched Polydendrons: a New Controlled Macromolecular Architecture with Self-Assembly in Water and Organic Solvents. Chem. Sci. 2014, 5, 1844-1853. 
36. Li, Z.; Dormidontova, E. E. Kinetics of Diblock Copolymer Micellization by Dissipative Particle Dynamics. Macromolecules 2010, 43, 3521-3531.

37. Dormidontova, E. E. Micellization Kinetics in Block Copolymer Solutions: Scaling Model. Macromolecules 1999, 32, (22), 7630-7644.

38. Zakharov, A. I.; Adzhemyan, L. T.; Shchekin, A. K. Relaxation Times and Modes of Disturbed Aggregate Distribution in Micellar Solutions with Fusion and Fission of Micelles. J. Chem. Phys. 2015, 143, 124902.

39. Kelley, E. G.; Murphy, R. P.; Seppala, J. E.; Smart, T. P.; Hann, S. D.; Sullivan, M. O.; Epps, T. H. Size Evolution of Highly Amphiphilic Macromolecular Solution Assemblies via a Distinct Bimodal Pathway. Nat. Commun. 2014, 5, 3599.

40. Zhang, J.; Xu, J.; Liu, S. Chain-Length Dependence of Diblock Copolymer Micellization Kinetics Studied by Stopped-Flow pH-Jump. J. Phys. Chem. B 2008, 112, 11284-11291.

41. Cheng, H.; Yuan, X.; Sun, X.; Li, K.; Zhou, Y.; Yan, D. Effect of Degree of Branching on the Self-Assembly of Amphiphilic Hyperbranched Multiarm Copolymers. Macromolecules 2010, $43,1143-1147$. 